\title{
Radiative seesaw dark matter
}

\author{
Ernest Ma \\ Department of Physics and Astronomy, University of California, Riverside, California 92521, USA \\ Valentina De Romeri \\ Institut de Física Corpuscular CSIC/Universitat de València, \\ Parc Científic de Paterna C/ Catedrático José Beltrán, 2 E-46980 Paterna, Valencia, Spain
}

(Received 6 May 2021; accepted 12 August 2021; published 7 September 2021)

\begin{abstract}
The singlet Majoron model of seesaw neutrino mass is appended by one dark Majorana fermion singlet $\chi$ with $L=2$ and one dark complex scalar singlet $\zeta$ with $L=1$. This simple setup allows $\chi$ to obtain a small radiative mass anchored by the same heavy right-handed neutrinos, whereas the one-loop decay of the standard model Higgs boson to $\chi \chi+\bar{\chi} \bar{\chi}$ provides the freeze-in mechanism for $\chi$ to be the light dark matter of the Universe.
\end{abstract}

DOI: 10.1103/PhysRevD.104.055004

\section{INTRODUCTION}

Neutrino mass [1] and dark matter [2] are two outstanding issues in particle physics and astrophysics. They may be essentially related, such as in scotogenic models [3], where the former requires the existence of the latter. They may also be indirectly related through lepton parity [4] or lepton number [5].

Dark matter is conventionally believed to be a massive particle of order $100 \mathrm{GeV}$ and interacts weakly with matter. Its annihilation cross section $\times$ relative velocity at rest should be about $3 \times 10^{-26} \mathrm{~cm}^{3} / \mathrm{s}$, as it freezes out of thermal equilibrium in the early Universe. It should then be observable in direct search experiments in underground laboratories.

An alternative is the freeze-in mechanism [6], where the dark matter interacts very weakly and slowly builds up its relic abundance from the decay of a massive particle, for example, before the latter itself goes out of thermal equilibrium. In this case, the direct detection of dark matter in underground experiments becomes very difficult, which is consistent with the mostly null results obtained so far.

The standard model (SM) of particle interactions conserves baryon number $B$ and $L$ automatically. If a singlet right-handed fermion $N_{R}$ is added, the term $\bar{N}_{R}\left(\nu_{L} \phi^{0}-e_{L} \phi^{+}\right)$is allowed by gauge invariance; hence, $N_{R}$ is naturally assigned $L=1$. On the other hand, gauge invariance also allows the $N_{R} N_{R}$ Majorana mass term.

Published by the American Physical Society under the terms of the Creative Commons Attribution 4.0 International license. Further distribution of this work must maintain attribution to the author(s) and the published article's title, journal citation, and DOI. Funded by SCOAP ${ }^{3}$.
Hence, $L$ naturally breaks to $(-1)^{L}$, and a seesaw mass for $\nu_{L}$ is obtained as is well known. Instead of letting gauge invariance decide on all the global and discrete symmetries of the Lagrangian, the latter may be imposed as additional inputs. In that case, other choices for $N_{R}$ are also possible [7].

Returning to the canonical choice of $L=1$ for $N_{R}$, if a new complex neutral scalar singlet $\eta$ with $L=2$ is added, then the term $\eta^{*} N_{R} N_{R}$ is allowed, and the Lagrangian with this term replacing the $N_{R} N_{R}$ term is invariant under the global symmetry $U(1)_{L}$. This conscious choice of a new particle with a nonzero lepton number was first made 40 years ago [8], where $\eta$ is assumed to have a nonzero vacuum expectation value, thereby breaking $U(1)_{L}$ spontaneously again to $(-1)^{L}$ but also with the appearance of a massless Goldstone boson called the singlet Majoron. The seesaw mechanism applies as before.

In this paper, the conscious choice of two additional particles with a nonzero lepton number is made. $\zeta$ is a neutral complex scalar with $L=1$, and $\chi_{L}$ is a neutral Majorana fermion with $L=2$. As expected [4] from $(-1)^{L+2 j}$ ( $j$ is the intrinsic spin of the particle), they are particles of odd dark parity. It is shown that $\chi_{L}$ obtains a small radiative mass anchored by $N_{R}$, and it becomes dark matter through the freeze-in mechanism from Higgs decay.

\section{DESCRIPTION OF MODEL}

The SM is extended first by three singlet right-handed neutrinos $N_{R}$ with lepton number $L=1$ as well as one complex scalar $\eta$ with $L=2$, so that the term $\eta^{*} N_{R} N_{R}$ appears in the Lagrangian, which is assumed invariant under $U(1)_{L}$. However, $\langle\eta\rangle \neq 0$ breaks $U(1)_{L}$ spontaneously, resulting in a singlet Majoron [8]. This well-known 
model is now extended to include one complex scalar $\zeta$ with $L=1$, and one Majorana fermion $\chi_{L}$ with $L=2$. The Lagrangian of the SM is then expanded, having

$$
\begin{aligned}
\mathcal{L}_{Y}= & -\frac{1}{2} f_{N} \eta^{*} N_{R} N_{R}-f_{\nu} \bar{N}_{R}\left(\nu_{L} \phi^{0}-l_{L} \phi^{+}\right) \\
& -f_{\chi} \bar{\chi}_{L} N_{R} \zeta+\text { H.c. },
\end{aligned}
$$

as well as

$$
\begin{aligned}
V(\zeta, \eta, \Phi)= & -\mu_{0}^{2} \Phi^{\dagger} \Phi-\mu_{2}^{2} \eta^{*} \eta+m_{1}^{2} \zeta^{*} \zeta+\frac{1}{2} \mu_{1} \eta^{*} \zeta^{2}+\text { H.c. } \\
& +\frac{1}{2} \lambda_{0}\left(\Phi^{\dagger} \Phi\right)^{2}+\frac{1}{2} \lambda_{1}\left(\zeta^{*} \zeta\right)^{2}+\frac{1}{2} \lambda_{2}\left(\eta^{*} \eta\right)^{2} \\
& +\lambda_{01}\left(\zeta^{*} \zeta\right)\left(\Phi^{\dagger} \Phi\right)+\lambda_{02}\left(\eta^{*} \eta\right)\left(\Phi^{\dagger} \Phi\right) \\
& +\lambda_{12}\left(\zeta^{*} \zeta\right)\left(\eta^{*} \eta\right) .
\end{aligned}
$$

In the above, lepton number $L$ is conserved, so that $\chi$ has no mass, and the $\eta^{2}$ and $\zeta^{2}$ terms are forbidden. However, it is spontaneously broken to lepton parity $(-1)^{L}$ by $\langle\eta\rangle$. Note that the $f_{N}$ term justifies the assignment of $L=2$ to $\eta$. The $\mu_{1}$ term justifies the assignment of $L=1$ to $\zeta$, and the $f_{\chi}$ term justifies the assignment of $L=2$ to $\chi_{L}$. Without $\eta$, the separate assignments of $L$ to $\zeta$ and $\chi_{L}$ would have been ambiguous. Let

$$
\begin{aligned}
\Phi & =\left(\begin{array}{c}
\phi^{+} \\
\phi^{0}
\end{array}\right)=\left(\begin{array}{c}
0 \\
(v+h) / \sqrt{2}
\end{array}\right), \\
\eta & =\left(u+\frac{\rho}{\sqrt{2}}\right) e^{i \theta / u \sqrt{2}}, \quad \zeta=\frac{1}{\sqrt{2}}\left(\zeta_{R}+i \zeta_{I}\right) e^{i \theta / 2 u \sqrt{2}},
\end{aligned}
$$

then $\eta^{*} \zeta^{2}+$ H.c. $=(u+\rho / \sqrt{2})\left(\zeta_{R}^{2}-\zeta_{I}^{2}\right)$. The minimum of $V$ is determined by

$$
\begin{aligned}
& \mu_{0}^{2}=\frac{1}{2} \lambda_{0} v^{2}+\lambda_{02} u^{2}, \\
& \mu_{2}^{2}=\lambda_{2} u^{2}+\frac{1}{2} \lambda_{02} v^{2} .
\end{aligned}
$$

As a result, the mass-squared matrix spanning $h$ and $\rho$ is given by

$$
\mathcal{M}_{h \rho}^{2}=\left(\begin{array}{cc}
\lambda_{0} v^{2} & \sqrt{2} \lambda_{02} v u \\
\sqrt{2} \lambda_{02} v u & 2 \lambda_{2} u^{2}
\end{array}\right) .
$$

Now, the $N_{R}$ mass is $m_{N}=f_{N} u$; hence, $u$ should be much greater than $v$. This means that $h$ is predominantly the SM Higgs boson and mixes very little with the very heavy $\rho$. As for the $\zeta_{R, I}$ masses, they are split by the $\mu_{1}$ term, i.e.,

$$
\begin{aligned}
& m_{R}^{2}=m_{1}^{2}+\frac{1}{2} \lambda_{01} v^{2}+\lambda_{12} u^{2}+\mu_{1} u, \\
& m_{I}^{2}=m_{1}^{2}+\frac{1}{2} \lambda_{01} v^{2}+\lambda_{12} u^{2}-\mu_{1} u .
\end{aligned}
$$

The neutrinos obtain canonical seesaw masses $m_{\nu}=m_{D}^{2} / m_{N}$, where $m_{D}=f_{\nu} v / \sqrt{2}$. Note that $\zeta$ is assumed not to have any vacuum expectation value by choosing $m_{1}^{2}>0$. If it does, then it will mix with $\Phi$ and $\eta$ through the $\lambda_{01}$ and $\lambda_{12}$ terms of Eq. (2). This would invalidate the assignment of dark parity that is discussed in the next section.

\section{LIGHT DARK MAJORANA FERMION}

The $\bar{\chi}_{L} N_{R} \zeta$ term is similar to the one found in the prototype renormalizable model [9] of fermionic dark matter $\chi_{L}$ supplemented by a real scalar counterpart $S$, i.e., $\bar{\chi}_{L} N_{R} S$. In that case, odd dark parity is imposed on $\chi$ and $S$. However, if lepton parity $L_{P}$ is used, i.e., even for $\chi$ and odd for $S, N$, the same dark parity $D_{P}$ is derived [4], namely, $D_{P}=L_{P}(-1)^{2 j}$. Note that $\chi$ is allowed an arbitrary Majorana mass. This scenario admits either $\chi$ or $S$ to be dark matter and has been studied extensively.

In the present model, $L$ is conserved in the Lagrangian; hence, $\chi$ (with $L=2$ ) is massless at tree level. After $L$ is spontaneously broken by two units, $\chi$ then obtains a radiative seesaw mass as shown in Fig. 1. Its structure is analogous to that of the scotogenic model [3], with its finiteness coming from the cancellation of the $\zeta_{R, I}$ contributions. Both $\chi$ and $\zeta_{R, I}$ belong to the dark sector, with odd dark parity $D_{P}$. The one-loop diagram of Fig. 1 is easily calculated,

$$
m_{\chi}=\frac{f_{\chi}^{2} m_{N}}{32 \pi^{2}}\left[\frac{m_{R}^{2} \ln \left(m_{R}^{2} / m_{N}^{2}\right)}{m_{R}^{2}-m_{N}^{2}}-\frac{m_{I}^{2} \ln \left(m_{I}^{2} / m_{N}^{2}\right)}{m_{I}^{2}-m_{N}^{2}}\right] .
$$

For $m_{R}^{2}-m_{I}^{2}=2 \mu_{1} u \ll m_{\zeta}^{2}=\left(m_{R}^{2}+m_{I}^{2}\right) / 2 \ll m_{N}^{2}$, this becomes

$$
m_{\chi}=\frac{f_{\chi}^{2} \mu_{1} u}{16 \pi^{2} m_{N}}\left[\ln \frac{m_{N}^{2}}{m_{\zeta}^{2}}-1\right] .
$$

Note that both $m_{\nu}$ and $m_{\chi}$ are of the seesaw form, anchored by $m_{N}$. However, $m_{\chi}$ can actually be large $[\mathcal{O}(\mathrm{GeV})$ or

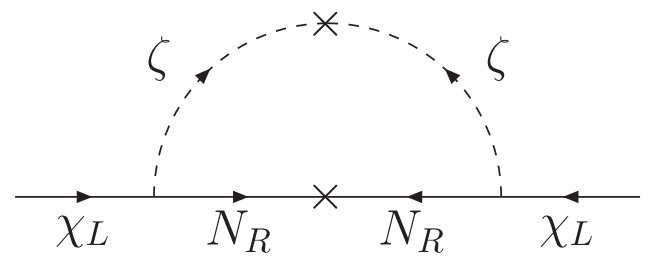

FIG. 1. One-loop radiative Majorana mass for the dark fermion $\chi$. 


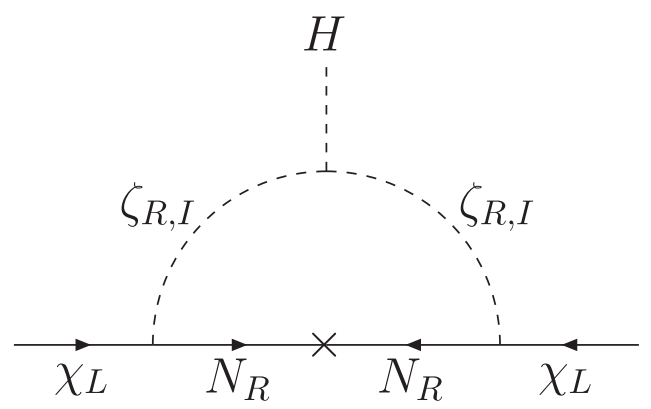

FIG. 2. Direct one-loop diagram for Higgs decay to $\chi \chi$.

larger], as it is also controlled by the free parameter $\mu_{1}$. A tree-level seesaw for light fermion dark matter is also possible in the context of $U(1)_{\chi}[10,11]$.

\section{PRODUCTION OF LIGHT FERMION DARK MATTER}

The only interaction involving $\chi$ is the $f_{\chi} \bar{\chi}_{L} N_{R} \zeta$ term. Assuming that the reheat temperature of the Universe is low enough (say a few $\mathrm{TeV}$ ), its relic abundance comes from freeze-in through Higgs decay, with the effective interaction

$$
\mathcal{L}_{\text {int }}=\frac{1}{2} f_{h} h(\chi \chi+\bar{\chi} \bar{\chi})
$$

where $f_{h}$ is given by Fig. 2,

$$
\begin{aligned}
f_{h}= & \frac{\lambda_{01} v f_{\chi}^{2} m_{N}}{32 \pi^{2}}\left[\frac{1}{m_{R}^{2}-m_{N}^{2}}-\frac{m_{N}^{2} \ln \left(m_{R}^{2} / m_{N}^{2}\right)}{\left(m_{R}^{2}-m_{N}^{2}\right)^{2}}-\frac{1}{m_{I}^{2}-m_{N}^{2}}\right. \\
& \left.+\frac{m_{N}^{2} \ln \left(m_{I}^{2} / m_{N}^{2}\right)}{\left(m_{I}^{2}-m_{N}^{2}\right)^{2}}\right] \\
= & \frac{-\lambda_{01} v f_{\chi}^{2} \mu_{1} u}{16 \pi^{2} m_{N} m_{\zeta}^{2}}=\frac{-\lambda_{01} v m_{\chi}}{m_{\zeta}^{2}}\left[\ln \frac{m_{N}^{2}}{m_{\zeta}^{2}}-1\right]^{-1}
\end{aligned}
$$

The decay rate of $h$ to $\chi \chi+\bar{\chi} \bar{\chi}$ is

$$
\Gamma_{h}=\frac{f_{h}^{2} m_{h}}{32 \pi} \sqrt{1-4 x^{2}}\left(1-2 x^{2}\right)
$$

where $x=m_{\chi} / m_{h}$.

Another one-loop diagram for $h$ decay to $\chi \chi$ is shown in Fig. 3. The corresponding effective coupling is given by

$$
f_{h}^{\prime}=\frac{\lambda_{01} v m_{\chi} f_{\chi}^{2}}{16 \pi^{2} m_{N}^{2}}\left[\ln \frac{m_{N}^{2}}{m_{\zeta}^{2}}-\frac{3}{2}\right]
$$

Hence, $\left|f_{h}^{\prime}\right| \ll\left|f_{h}\right|$ for $m_{\zeta} \ll m_{N}$.

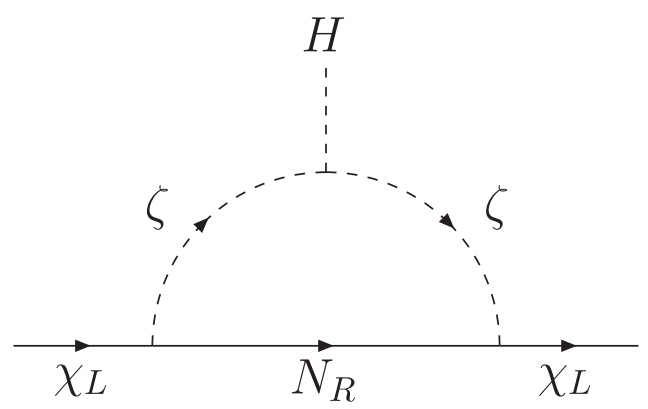

FIG. 3. One-loop diagram for Higgs decay to $\chi \chi$ through external $m_{\chi}$ insertion.

\section{PHENOMENOLOGY OF FERMION DARK MATTER}

We assume that the reheat temperature of the Universe after inflation is below the decoupling temperature of $\chi$ but above $m_{H}$, say $T_{R} \sim 1-10 \mathrm{TeV}$. In such a scenario, $\chi$ is a feebly interacting massive particle (FIMP), which only production mechanism is freeze-in $[6,12]$ through Higgs decay, before the latter decouples from the thermal bath. (In principle, there could also be $2 \rightarrow 2$ scattering of $\chi$ with other states in the thermal bath, mediated by the Higgs boson $[13,14]$.$) The relic abundance of \chi$, initially negligible in the early Universe, gradually grows via its feeble coupling $f_{h}$. Both $N_{R}$ and $\zeta$ are taken to be much heavier than $T_{R}$ so that their abundances are Boltzmann suppressed. On the contrary, if $m_{N}$ or $m_{\zeta} \lesssim T_{R}$, new interaction channels would open, and the relic density of $\chi$ could excessively increase due to the term $f_{\chi} \bar{\chi}_{L} N_{R} \zeta$, such as $N_{R} \bar{N}_{R} \rightarrow \chi \bar{\chi}, \zeta \zeta \rightarrow \chi \chi$, or the decays of $\zeta$. These processes could possibly lead to the thermalization of $\chi$. Even if $m_{N}\left(m_{\zeta}\right) \gg T_{R}$, the channel $\nu \bar{\nu} \rightarrow \chi \bar{\chi}$ is always present, although it is suppressed by the mixing of $N_{R}$ with the light active neutrinos. Eventually, we find that this channel never contributes sizeably to the thermalization of $\chi$.

We perform a scan varying randomly the main free parameters which characterize the model, and we rely on public computer tools to 1) implement the model with (FeynRules 2.0 [15] and CalcHEP 3.4 [16]) and 2) numerically compute the relic abundance of $\chi$ via freeze-in with (Micromegas 5.2.4 [17]). In the analysis, we require the parameters to comply with the hierarchy of scales $2 \mu_{1} u \ll m_{\zeta}^{2} \ll m_{N}^{2}$ and with light neutrino mass constraints.

Figure 4 shows the values of $f_{h}$ required to obtain the observed relic density, as a function of $m_{\chi}$. The green plain line denotes the contour along which $\Omega h^{2}=0.12$ [18]. Solutions in the green shaded area are excluded as they lead to overabundant dark matter. Values in the white area are allowed, but in this case, another dark matter candidate would be required. As expected in a FIMP scenario, for the relic abundance of $\chi$ to meet current observations, $f_{h}$ must be small: $f_{h} \lesssim 3 \times 10^{-11}$ for $m_{\chi} \sim 0.1 \mathrm{GeV}$. Its smallness is 


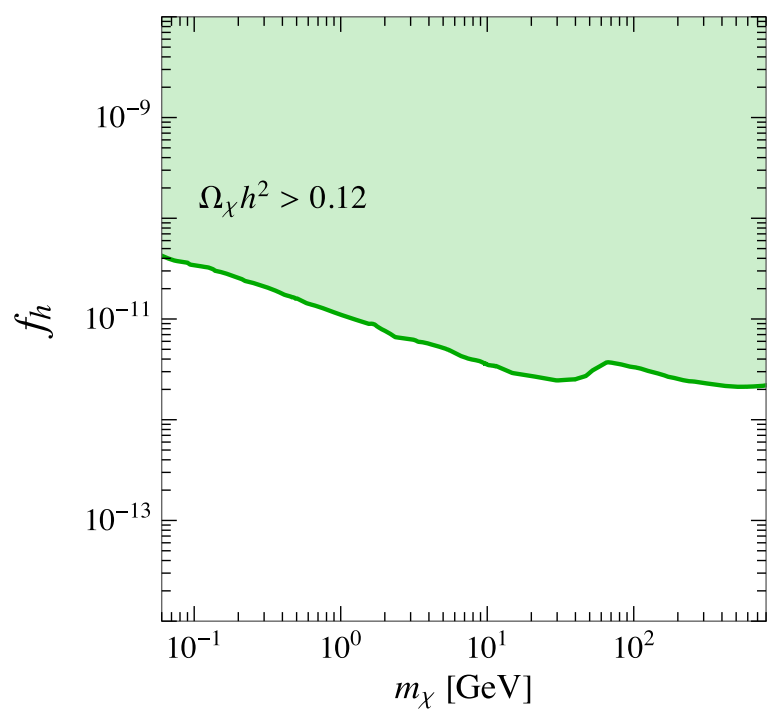

FIG. 4. $f_{h}$ as a function of $m_{\chi}$. The green plain line denotes the contour along which $\Omega h^{2}=0.12$ [18]. The region in the green shaded area is excluded as it leads to overabundant dark matter.

here driven by the mass hierarchy $m_{\chi} \ll v \ll m_{\zeta}$ and in a minor part by its loop nature. Let us notice that the abundance of $\chi$ is insensitive to $T_{R}$ as long as $T_{R} \ll m_{\zeta}, m_{N_{R}}$. Let us recall that this last assumption is required in order to prevent the thermalization of $\chi$ and thus justifies our choice of $T_{R} \sim 1-10 \mathrm{TeV}$ for the benchmark values and mass hierarchies here considered.

For the sake of example, we summarize in Table I the values of the relevant parameters corresponding to few benchmark points which lead to a relic density compatible with current observations.

Finally, Fig. 5 depicts the result of a numerical scan of the model parameter space, including the benchmark points collected in Table I. Blue points denote solutions which are compatible with current cosmological observations, while gray points are excluded because of $\Omega h^{2}>0.12$.

\section{CONSTRAINTS ON THE MAJORON}

The massless singlet Majoron $\theta$, which couples primarily to the light active neutrinos with a strength suppressed by

TABLE I. Benchmark points which lead to a relic density of $\chi$ compatible with current observations.

\begin{tabular}{lrccll}
\hline \hline$m_{\chi}[\mathrm{GeV}]$ & $m_{N}[\mathrm{GeV}]$ & $m_{\zeta}[\mathrm{GeV}]$ & $f_{h}$ & \multicolumn{1}{c}{$f_{\chi}$} & $\Omega h^{2}$ \\
\hline 0.11 & $1.4 \times 10^{7}$ & $7.34 \times 10^{4}$ & $3.26 \times 10^{-11}$ & 1 & 0.10 \\
1.13 & $1.19 \times 10^{6}$ & $4.47 \times 10^{5}$ & $1.02 \times 10^{-11}$ & 0.24 & 0.11 \\
2.42 & $1.6 \times 10^{5}$ & $3.57 \times 10^{4}$ & $7.54 \times 10^{-12}$ & 3.7 & 0.12 \\
21.14 & $8.3 \times 10^{5}$ & $1.21 \times 10^{5}$ & $2.46 \times 10^{-12}$ & 7.19 & 0.098 \\
\hline \hline
\end{tabular}

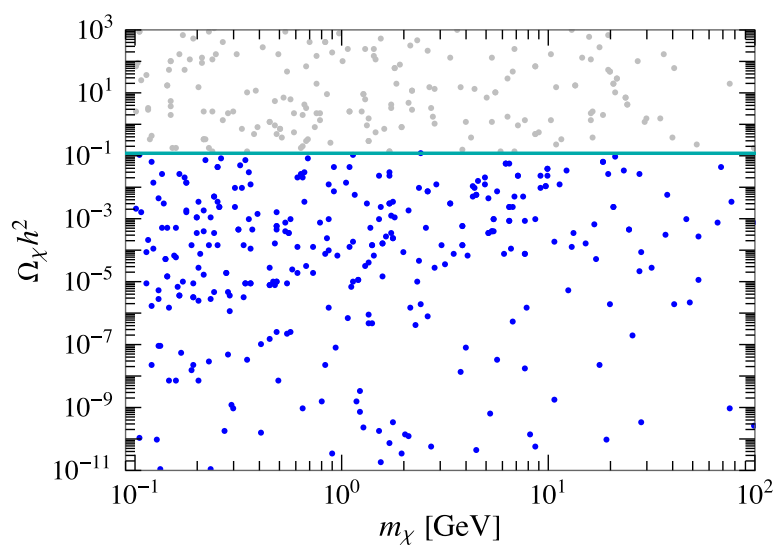

FIG. 5. $\Omega h^{2}$ as a function of $m_{\chi}$. The dark cyan line denotes the observed value $\Omega h^{2}=0.12$ [18].

powers of $u$, may play a role in cosmological and astrophysical environments. A single thermally decoupled massless Majoron can contribute to the effective number of neutrinos, $\Delta N_{\text {eff }}$. This extra contribution to the radiation density depends on the Majoron freeze-out temperature. However, because of the large values of the lepton number symmetry breaking scale here considered $(u \gtrsim 10 \mathrm{TeV})$ and the low reheat temperature, we find that the massless Majoron never thermalizes with the thermal bath. Still, it could be produced via freeze-in through its linear coupling with the light active neutrinos. Also in this case, given the large values of $u$, the corresponding neutrino-Majoron couplings lie below current cosmological constraints (see, e.g., [19]). Finally, laboratory searches for nonobserved lepton flavor violating rare decays (e.g., $\ell \rightarrow \ell^{\prime} \theta$ ) together with stellar cooling bounds further constrain the Majoron dimensionless parameters $m_{D}^{2} / v u \lesssim 10^{-5}-10^{-6}$ [20]. As before, since $u$ is much heavier than $v$ and $f_{\nu}$ can be quite small, the solutions considered in our numerical scan always fall below the current constraints.

\section{CONCLUDING REMARKS}

It is proposed that the lepton number may be a key to understanding light freeze-in dark matter. In the context of a spontaneously broken lepton number, first pointed out 40 years ago with a scalar having $L=2$, it is proposed that two other particles exist with a nonzero lepton number, a scalar $\zeta$ with $L=1$ and a Majorana fermion $\chi$ with $L=2$. It is shown that $\chi$ may acquire a small radiative seesaw mass and becomes freeze-in dark matter from the decay of the SM Higgs boson. However, this proposal is similar to others in its practical predictions (see, e.g., [21]), it has the advantage of explaining the smallness of the dark matter mass based on a symmetry, i.e., lepton number, as well as the weakness of its interaction. 


\section{ACKNOWLEDGMENTS}

This work was supported in part by the U.S. Department of Energy Grant No. DE-SC0008541. V. D. R. acknowledges financial support by Grant No. SEJI/2020/016 (project "Les Fosques") funded by Generalitat Valenciana, by the Universitat de València through the subprogramme "ATRACCIÓ DE TALENT 2019" and partial support by the Spanish Grant No. FPA2017-85216-P (AEI/FEDER, UE).

[1] See for example A. de Gouvea, Annu. Rev. Nucl. Part. Sci. 66, 197 (2016).

[2] See for example B.-L. Young, Front. Phys. 12, 121201 (2017).

[3] E. Ma, Phys. Rev. D 73, 077301 (2006).

[4] E. Ma, Phys. Rev. Lett. 115, 011801 (2015).

[5] E. Ma, Phys. Lett. B 809, 135736 (2020).

[6] L. J. Hall, K. Jedamzik, J. March-Russell, and S. M. West, J. High Energy Phys. 03 (2010) 080.

[7] E. Ma, Mod. Phys. Lett. A 32, 173007 (2017).

[8] Y. Chikashige, R. N. Mohapatra, and R. D. Peccei, Phys. Lett. 98B, 265 (1981).

[9] M. Pospelov, A. Ritz, and M. B. Voloshin, Phys. Lett. B 662, 53 (2008).

[10] E. Ma, Phys. Rev. D 98, 091701(R) (2018).

[11] E. Ma, Lett. High Energy Phys. 2, 103 (2019).

[12] J. McDonald, Phys. Rev. Lett. 88, 091304 (2002).
[13] G. Arcadi and L. Covi, J. Cosmol. Astropart. Phys. 08 (2013) 005.

[14] M. Blennow, E. Fernandez-Martinez, and B. Zaldivar, J. Cosmol. Astropart. Phys. 01 (2014) 003.

[15] A. Alloul, N. D. Christensen, C. Degrande, C. Duhr, and B. Fuks, Comput. Phys. Commun. 185, 2250 (2014).

[16] A. Belyaev, N. D. Christensen, and A. Pukhov, Comput. Phys. Commun. 184, 1729 (2013).

[17] G. Bélanger, F. Boudjema, A. Goudelis, A. Pukhov, and B. Zaldivar, Comput. Phys. Commun. 231, 173 (2018).

[18] N. Aghanim et al. (Planck Collaboration), Astron. Astrophys. 641, A6 (2020).

[19] D. Baumann, D. Green, and B. Wallisch, Phys. Rev. Lett. 117, 171301 (2016).

[20] J. Heeck and H. H. Patel, Phys. Rev. D 100, 095015 (2019).

[21] E. Molinaro, C. E. Yaguna, and O. Zapata, J. Cosmol. Astropart. Phys. 07 (2014) 015. 